\title{
Narrow band imaging (NBI) cystoscopy and assisted bipolar TURBT: A preliminary experience in a single centre
}

\author{
Roberto Giulianelli, Barbara Cristina Gentile, Luca Albanesi, Paola Tariciotti, Gabriella Mirabile \\ C.Ur.A., Urology Department, Nuova Villa Claudia Clinic, Rome.
}

\begin{abstract}
Summary Objective: The aim of this study was to compare, in order to increase our ability to detect bladder cancer, the predictive power of narrow band imaging (NBI) versus white light cystoscopy (WL).

The secondary objective was to evaluate how the preoperative use of NBI cystoscopy can increase the ability to detect bladder lesions in terms of status, multi-focality and dimensions. Materials and methods: Between June 2010 and April 2012, 797 consecutive patients, 423 male and 374 female, affected by suspected bladder cancer lesions, underwent to WL plus NBI cystoscopy and subsequently to WL Bipolar Gyrus PK (Olympus, Tokyo, Japan) transurethral resection of bladder tumour (WL-TURBT). The average follow-up was 24 (16-38) months. Mean age was 67.7 yrs. (range 46-88).
\end{abstract}

All the patients underwent by same surgeon to $\mathrm{WL}$ resection (WL-TURBT) of the previously identified lesions by same surgeon. All the removed tissue was sent separately for histological evaluation after mapping the areas of resection on a topographic sheet.

Results: In our study we considered 797 patients that matched our inclusion criteria. Through the use of WL cystoscopy, we identified 603 patients $(75.53 \%)$ with suspicious lesions, instead, with the use of light NBI, we found 786 patients with suspicious lesions (98.49\%). The use of NBI cystoscopy increases by approximately $30 \%$ the specific ability to detect lesions not otherwise visible with WL cystoscopy (OR 21.9 and RR 1.30), in particular for patients with lesions size $<3 \mathrm{~cm}$ (OR 24.00; RR 1.40), unifocal (OR: 22.28; RR 1.47) and recurrent (OR 58.4; RR 1.34). Pathology demonstrated the presence of cancer in 512 (64.2\%) patients, of whom $412(51.8 \%)$ were visible both with WL cystoscopy and NBI cystoscopy.

In our experience, only $11(1.38 \%)$ lesions were only positive at WL cystoscopy (negative at NBI cystoscopy) thus 501 (62.8\%, OR 10.13; RR 1.21) patients showed bladder oncological lesions positive at NBI cystoscopy. In these patients, the use the NBI Cystoscopy has better highlighted a recurrence $(p<0.005$; OR 22.8, RR 1.23; 95\% CI-1.13 to 0.24) or a lesion < $3 \mathrm{~cm}$ ( $p<0.05$; OR 11.4, RR 1.30; 95\% CI-0.18 to $0.29)$ or a unifocal lesion $(p<0.005$; OR 10.38, RR 1.34, CI 0.18 to 0.30 ).

Conclusions: The use of NBI cystoscopy, significantly increases by approximately $30 \%$ our predictive power to identify neoplastic lesions, especially unifocal or $<3 \mathrm{~cm}$ or recurrent lesions. Following WLTURBT, stage, dimension and focaliity are statistically significant determinants $(p<0.001)$ of the bladder oncological lesions detected by NBI cystoscopy rather than by WL cystoscopy.

KEY WORDS: Bladder cancer; Narrow band imaging (NBI); Cystoscopy; Bipolar TURBT.

Submitted 15 August 2017; Accepted 18 August 2017

\section{INTRODUCTION}

Bladder cancer is a very common malignant genitourinary tumour and transitional cell carcinoma accounts for almost $90 \%$ of all primary bladder tumours. The standard method used to diagnose bladder cancer and monitor patients in the follow-up is white light (WL) cystoscopy. Unfortunately, this method may not be able to detect small papillary lesions and carcinoma in situ (CIS) lesions (1). Tumours not detected in the course of TURBT will appear later as a relapse, and some of them may become invasive. This calls for the development of better endoscopic methods, namely fluorescence (2-4), and narrow banding imaging (NBI) cystoscopy (5). NBI is a technique for improving optical images. It is designed for endoscopy and its purpose is to increase the contrast between mucosal surfaces and microvascular structures without resorting to dyes. This technique is based on a phenomenon by which the depth of light penetration into the mucosa increases as the wavelength increases. In NBI, the tissue surface is illuminated with narrow band light, with wavelengths in the blue and green light spectra $(415 \mathrm{~nm}$ and $540 \mathrm{~nm}$ respectively) (6). The primary objective of this study was to assess the ability of NBI to increase the detection rate of lesions not visible with WL cystoscopy. The secondary objective was to evaluate how the preoperative use of NBI cystoscopy can increase the ability to detect bladder lesions in terms of status, focality and dimensions after TURBT, as opposed to WL cystoscopy.

\section{Materials AND Methods}

From June 2010 to April 2012, 797 consecutive patients (pts.), 423 male and 374 female, affected by primary (461 pts, 57.8\%), recurrent (336 pts, 42.1\%), unifocal (491 pts., 61.6\%), multifocals (306 pts., $38.3 \%$ ), $<3 \mathrm{~cm}$ (570 pts., $71.5 \%$ ) and $>3 \mathrm{~cm}$ (227 pts., $28.4 \%$ ) suspicious non-muscle invasive bladder tumours, underwent WL plus NBI cystoscopy and WL plus NBI Bipolar TURBT with a Bipolar Gyrus PK Scalpel in saline (TURis). The mean follow-up was 24 (16-38) month. Indication of suitability for TURBt was provided on the basis of the EAU Guideline 2010. All patients provided written informed consent prior to the study.

All procedures were carried out initially by performing a cystoscopy with white light. The characterization of the sites, including the number, size and appearance of the neoplasms, were recorded on a topographic bladder map. 
Subsequently a cystoscopy with NBI was carried out to confirm what had been seen by white light examination, and to report other suspicious areas at NBI light. These, too, were recorded on the topographic bladder map. All endoscopic resections were performed with an Gyrus PK scalpel, bipolar generator (Olympus, Tokyo, Japan), in saline, with optics at 30 degrees. Resection of each lesion was carried out with white light by the same surgeon who had performed cystoscopy and all histopathological evaluations were performed by the a single pathologist on the basis of 2004 WHO classification.

In order to evaluate the efficacy of NBI vs WL cystoscopy, we conducted a hypothesis test to determine whether the difference between the two proportions of positive results obtained by WL and by NBI (p_wl and p_nbi) was significant. The two-proportion z-test was administered on the overall sample and, after TURBT, on the hystologically positive set of patients. The appropriate test assumes a model for matched pairs (7). The analysis was carried out in relation to status (primitive and recurring), focality and dimension of detected lesions. All Z-scores (before and after TURBT) were largely below the selected significance level $(\alpha=0.05)$.

The related confidence intervals were also calculated. In addition we estimated odds ratio (OR) and relative risk (RR) to quantify how strong is the difference between NBI and WL results. Logistic regression was applied to predict which lesions were in fact malignant after NBI cystoscopy. Multiple linear regression analyses tested the association between different prognostic variables and their impact on the ability to identify bladder lesions following NBI cystoscopy.

\section{Results}

In our study we considered 797 patients that matched our inclusion criteria. WL cystoscopy was used to identify 603 patients (75.5\%) with suspicious lesions, while the use of NBI following WL allowed identifying a total of 786 patients $(98.49 \%)$.

A total of 1.571 suspicious lesions were identified in 797 patients, some of whom had multifocal lesions. Of these lesions, 496 (50.6\%) were single lesions and 1.075 (49.3\%) multiple lesions. Of these 1,571 lesions, 1.337 (85.11\%) were identified by WL cystoscopy alone. The subsequent use of NBI light allowed finding 234 lesions (14.89\%), not visible otherwise with WL, reaching a total of 1.571 suspicious lesions. The use of NBI cystoscopy increases by approximately $30 \%$ the ability to detect lesions not otherwise visible with WL cystoscopy alone (OR 21.9 and RR 1.30), particularly in patients with lesion size $<3 \mathrm{~cm}$

Table 1.

Table 2.
WL and NBI cystoscopy results (before TURBT).

\begin{tabular}{|c|c|c|c|c|c|c|c|}
\hline All cases & Yes & No & Total & Odds & Odds ratio & Absolute risk & Relative risk \\
\hline NBI positive & 786 & 11 & 797 & 66.14 & & 0.985 & \\
\hline WL positive & 603 & 194 & 797 & 3.087 & 21.9 & 0.755 & 1.304 \\
\hline $\begin{array}{l}\text { Primitive } \\
\text { NBI positive } \\
\text { WL positive }\end{array}$ & $\begin{array}{l}452 \\
354\end{array}$ & $\begin{array}{c}9 \\
107\end{array}$ & $\begin{array}{l}461 \\
461\end{array}$ & $\begin{array}{r}45.10 \\
3.26\end{array}$ & 13.79 & $\begin{array}{l}0.978 \\
0.785\end{array}$ & 1.27 \\
\hline $\begin{array}{l}\text { Recurrent } \\
\text { NBI positive } \\
\text { WL positive }\end{array}$ & $\begin{array}{l}334 \\
249\end{array}$ & $\begin{array}{c}2 \\
87\end{array}$ & $\begin{array}{l}336 \\
336\end{array}$ & $\begin{array}{c}167.0 \\
2.86\end{array}$ & 55.34 & $\begin{array}{l}0.994 \\
0.741\end{array}$ & 1.341 \\
\hline $\begin{array}{l}\text { Unifocal } \\
\text { NBI positive } \\
\text { WL positive }\end{array}$ & $\begin{array}{l}481 \\
325\end{array}$ & $\begin{array}{c}10 \\
166\end{array}$ & $\begin{array}{l}491 \\
491\end{array}$ & $\begin{array}{l}43.6 \\
1.95\end{array}$ & 22.28 & $\begin{array}{l}0.978 \\
0.662\end{array}$ & 1.47 \\
\hline $\begin{array}{l}\text { Multifocal } \\
\text { NBI positive } \\
\text { WL positive }\end{array}$ & $\begin{array}{l}305 \\
277\end{array}$ & $\begin{array}{c}1 \\
29\end{array}$ & $\begin{array}{l}306 \\
306\end{array}$ & $\begin{array}{c}305.0 \\
9.55\end{array}$ & 31.93 & $\begin{array}{l}0.997 \\
0.905\end{array}$ & \\
\hline $\begin{array}{l}\mathbf{3} \mathbf{c m} \\
\text { NBI positive } \\
\text { WL positive }\end{array}$ & $\begin{array}{l}560 \\
399\end{array}$ & $\begin{array}{c}10 \\
171\end{array}$ & $\begin{array}{l}570 \\
570\end{array}$ & $\begin{array}{l}56.0 \\
2.33\end{array}$ & 24.00 & $\begin{array}{l}0.982 \\
0.700\end{array}$ & 1.404 \\
\hline $\begin{array}{l}\mathbf{3} \mathbf{~ c m} \\
\text { NBI positive } \\
\text { WL positive }\end{array}$ & $\begin{array}{l}226 \\
203\end{array}$ & $\begin{array}{c}1 \\
24\end{array}$ & $\begin{array}{l}227 \\
227\end{array}$ & $\begin{array}{l}22.6 \\
8.45\end{array}$ & 13.3 & $\begin{array}{l}0.991 \\
0.894\end{array}$ & 1.108 \\
\hline
\end{tabular}

(OR 24.00; RR 1.40), unifocal (OR: 22.28; RR 1.47) and recurrent lesions (OR 58.4; RR 1.34) (Table 1).

All 797 patients were subjected to bipolar TURBT with Gyrus PK (Olympus, Tokyo, Japan). The final histology results demonstrated the presence of cancer in 512 (64.2\%) patients (Table 2), of whom 412 (51.8\%) were visible both with WL cystoscopy and NBI cystoscopy. In our experience, 501 (62.8\%) patients showed bladder oncological lesions positive in NBI cystoscopy thus only $11(1.38 \%)$ lesions were only positive at WL cystoscopy. Totally, 1,571 cancer lesions were identified as suspicious, 1,051 lesions $(66.85 \%)$ were positive for malignancy and 520 (33.14\%) negative for malignancy.

The histology after WLTURBT showed the ability of NBI cystoscopy to identify, in more than $20 \%$, a lesion oncologically significant than was not evident at WL cystoscopy (Table 2).

WL and NBI cystoscopy results (hystologically positive after TURBT).

\begin{tabular}{|c|c|c|c|c|c|c|c|}
\hline All cases & Yes & No & Total & Odds & Odds ratio & Absolute risk & Relative risk \\
\hline NBI positive & 501 & 11 & 512 & 41.6 & & 0.977 & \\
\hline WL positive & 412 & 100 & 512 & 4.12 & 10.1 & 0.805 & 1.21 \\
\hline $\begin{array}{l}\text { Primitive } \\
\text { NBI positive } \\
\text { WL positive }\end{array}$ & $\begin{array}{l}317 \\
263\end{array}$ & $\begin{array}{c}9 \\
63\end{array}$ & $\begin{array}{l}326 \\
326\end{array}$ & $\begin{array}{c}31.6 \\
4.17\end{array}$ & 7.57 & $\begin{array}{l}0.969 \\
0.807\end{array}$ & 1.20 \\
\hline $\begin{array}{l}\text { Recurrent } \\
\text { NBI positive } \\
\text { WL positive }\end{array}$ & $\begin{array}{l}184 \\
149\end{array}$ & $\begin{array}{c}2 \\
37\end{array}$ & $\begin{array}{l}186 \\
186\end{array}$ & $\begin{array}{l}92.0 \\
4.02\end{array}$ & 22.84 & $\begin{array}{l}0.989 \\
0.801\end{array}$ & 1.235 \\
\hline $\begin{array}{l}\text { Unifocal } \\
\text { NBI positive } \\
\text { WL positive }\end{array}$ & $\begin{array}{l}290 \\
215\end{array}$ & $\begin{array}{l}10 \\
85\end{array}$ & $\begin{array}{l}300 \\
300\end{array}$ & $\begin{array}{r}26.2 \\
2.5\end{array}$ & 10.38 & $\begin{array}{l}0.963 \\
0.717\end{array}$ & 1.344 \\
\hline $\begin{array}{l}\text { Multifocal } \\
\text { NBI positive } \\
\text { WL positive }\end{array}$ & $\begin{array}{l}211 \\
197\end{array}$ & $\begin{array}{c}1 \\
15\end{array}$ & $\begin{array}{l}212 \\
212\end{array}$ & $\begin{array}{c}211.0 \\
13.3\end{array}$ & 16.06 & $\begin{array}{l}0.995 \\
0.929\end{array}$ & 1.071 \\
\hline $\begin{array}{l}<\mathbf{3 c m} \\
\text { NBI positive } \\
\text { WL positive }\end{array}$ & $\begin{array}{l}309 \\
223\end{array}$ & $\begin{array}{l}10 \\
85\end{array}$ & $\begin{array}{l}319 \\
319\end{array}$ & $\begin{array}{l}30.9 \\
2.70\end{array}$ & 11.40 & $\begin{array}{l}0.989 \\
0.730\end{array}$ & 1.326 \\
\hline
\end{tabular}


Thanks to use of the NBI light we identified 500 patients with lesions NBI positive oncologically significant (62.7\%; OR 10.13; RR 1.21). In particular, NBI cystoscopy improved bladder neoplasms detection rate of WL cystoscopy in recurrent (OR 22.8 vs 7.5; RR 1.23 vs 1.20), <3 $\mathrm{cm}$ (OR 11.4 vs 7.4; RR 1.30 vs 1.06) and unifocal tumours (OR 10.38 vs 16.06; RR 1.34 vs 1.07). The difference in detection rate is not so high as in recurrences in primitive lesions (RR 1.23 vs 1.20). In 512 patients with tumors detected after WL TURBT, the use the NBI cystoscopy has more frequently demonstrated a recurrent injury ( $p<0.005 ; 95 \%$ CI-1.13 to 0.24 ) or a lesion $<3 \mathrm{~cm}$ ( $p>0.05 ; 95 \%$ CI-0.18 to 0.29 ) or an unifocal lesion ( $p<$ 0.005 ; CI 0.18 to 0.30 ). It is clear that with the NBI cystoscopy, compared to WL cystoscopy alone, we could identify a statistically significantly greater number of neoplastic lesions, in relation to pathological stage and grade ( $p<0.005$, IC-95\% 0.13- 0.21 and $p<0.005$; 95\% CI0.14 to 0.24 , respectively). In particular, we highlighted an higher number of CIS lesions ( $p<0.005$, IC-95\% from 0.48 to 0.92 ) and HG lesions ( $p<0.005$; CI-95\%. In our experience we identified in the group of 512 patients with bladder cancer confirmed after WLTURBT, 88 patients who had bladder neoplastic lesions detected only through the use of NBI cystoscopy (WL cystoscopy negative) with an adjunctive detection rate (ADR) of $11.4 \%$.

The overall false positive detection rate was 33.14\% (521 lesions) and after NBI cystoscopy this rate was $39.44 \%$. Using the logistic model, we observed that the use of NBI cystoscopy significantly increases our predictive power to identify lesions not visible with WL cystoscopy: primitive lesions (95\% CI 2.97-1.095; p < 0.02), multifocal (95\% CI $0.06-012$; p < 0.0001), and < 3 cm (95\% CI 0.05-0.13; p $<0.001)$. It is interesting to note that HG (high-grade) neoplasms (95\% CI 0.14-0.24; $\mathrm{p}<0.001$ ) tend to reach a value very close to significance compared to papillary urothelial neoplasms of low malignant potential (PUNMP) (95\% CI 0.004-0.04; $\mathrm{p}<0.005)$ and low grade neoplasms (low-grade) (95\% CI 0.10-0.21; p < 0.001).

Using NBI cystoscopy, the likelihood of identifying patients with a primitive tumour, compared to patients with a relapse, reached approximately $80 \%$ (point estimate $1.790,95 \%$ CI 1095-2927). In a patient with a unifocal tumour, the likelihood of having a bladder tumour detected by NBI cystoscopy increases by about seven times compared with those a multifocal tumour (point estimate $0.153,95 \%$ CI 0.060-0.390).

NBI cystoscopy has also proved to be very useful in CIS. By comparing the sensitivity, specificity, positive predictive value and negative predictive value (NPV) of NBI versus WL cystoscopy for CIS lesions, we noticed that sensitivity and NPV were the statistically significant values $(100 \%, 95 \% \mathrm{CI}, \mathrm{p}<005$, and $80.62 \%$, 95\% CI, $100 \%, 95 \% \mathrm{CI}, \mathrm{p}<005$, and $78.35 \%$, respectively). NBI cystoscopy increases the likelihood of detecting a CIS lesion by about one and a half times compared to a patient with a pTa lesion $(\mathrm{p}<0.001)$.

\section{Discussion}

Bladder cancer is considered the most expensive tumour both in terms of costs for patients per year and in terms of operating costs per patient. Up to $70 \%$ of patients with NMIBC will develop a recurrence after TURBT (8).

Photodynamic diagnosis with Hexaminolevulinate blue light cystoscopy has made significant advances in terms of rates (9) of diagnostic accuracy and recurrence for the detection of CIS lesions (10), as well as pTa and pT1 tumours (11).

However, some questions have been raised as regards the cost-effectiveness of this approach.

Numerous studies have demonstrated the ability to display improved images of the surface layers of the various apparatuses using NBI $(10,12-18)$. NBI is a diagnostic imaging technique that improves the contrast between mucosal surfaces and microvascular structures, based on the wavelength-dependent increase in the depth of light penetration into the mucosa, without additional costs to conventional cystoscopy and without lengthening the operating time.

In our experience, using WL and NBI cystoscopy together, we have identified 234 lesions (14.1\%) that WL cystoscopy alone would not have otherwise been able to identify. However, the essential result is not so much the number of lesions that can be identified using NBI as opposed to the number of those visible with WL alone, but rather how many of these are neoplasms. Of these 234 lesions identified with NBI alone, as many as 127 were bladder tumours (12.17\%) in 88 patients $(11,4 \%)$. Comparing our experience with the data available in the literature, we were able to find some interesting data.

The experience of Bryan et al. (5) showed a greater capacity to identify lesions ( $\Delta=-7.15 \%$ ) compared to our results, while our technique showed an improvement $(\Delta=$ $+3.81 \%$ ) compared to the results of Herr et al. (19)

In fact, we detected $12.17 \%$ of lesions not otherwise detectable, by WL cystoscopy, thus placing our experience between the two aforementioned studies.

To better understand the diagnostic efficacy of the NBI compared to WL cystoscopy an analysis of the odds ratio was performed (positive results against the negatives for each type of investigation). The two methods of cystoscopy did not change the state of the patient on which they were performed. Therefore 797 cases can be considered as a sample to which two different but non-interacting methods were administered.

The odds ratio, measuring the frequency of success of a technique respect to another, in our case highlights the success of NBI. In fact a value greater than 1 indicates greater effectiveness of NBI in the detection of lesions against the non-detection. If we consider all the cases, the calculated value of $\mathrm{OR}=21.90$ (Table 1 ) shows a strong prevalence of NBI identification of lesions. A more intuitive interpretation of the results is made up of the relative risk. In fact, this index relates the absolute risk (ratio of successes and the total number of cases) of the two methods. In this way it is possible to assess with greater immediacy the effectiveness of the method NBI compared to WL.

The use of NBI cystoscopy has therefore allowed improving our ability to overall detect lesions not otherwise visible with WL cystoscopy alone $(\mathrm{ADR}=+30 \%)$ in terms of focality (unifocals: OR 22.28; RR 1.47) as well as dimensions < $3 \mathrm{~cm}$, OR 24.00; RR 1.40) and status (recurrences: OR 58.4; RR 1.34). 
Following WL TURBT, we observed 500 pts with NBI bladder oncological lesions, with a ADR of $20 \%$. The use of NBI cystoscopy, significantly increases our predictive power to identify lesions not visible with WL cystoscopy, especially for unifocal lesions ( $\mathrm{p}<0.005$, IC-95\%0.18$0.30)$, those $<3 \mathrm{~cm}(\mathrm{p}<0.005$, IC-95\% 0.18-0.29) and recurrent lesions ( $p<0.005$, IC-95\% 0.13-0.24).

Similarly, through the use of NBI light, we observed that, as regards pathologic stage and grade, we registered an overall increase in bladder lesion detection rate $(\mathrm{p}<$ 0.005 , IC-95\% 0.13-0.21 and $\mathrm{p}<0.005$; IC-95\% 0.140.24 , respectively).

In our experience, the use of NBI cystoscopy significantly increased the ability to detect both CIS lesions ( $\mathrm{p}<$ $0.0001)$ and HG ( $<$ < 0.005) (Table $\mathrm{C}$ : $\mathrm{d}-\mathrm{e}$ ) compared to $\mathrm{WL}$ cystoscopy. The relative risk (RR) to identify a bladder tumour with NBI TURBT is 3 times greater in the case of a pTa LG lesion and about 8 times greater if the lesion is a CIS. In our experience, as well as in Herr's experience (19), the use of NBI cystoscopy in comparison to WL cystoscopy was particularly useful in the identification of CIS lesions, showing a sensitivity and NPV of $100 \%$ vs. $80.62 \%$ and $100 \%$ vs. $78.35 \%$ ( $<<0.05$ ) respectively. By contrast, we recorded a non-significant specificity and PPV. In our experience, the overall false positive detection rate was $33.14 \%$ (521 lesions) and after NBI cystoscopy this rate amounted to $39.44 \%$. These data overlap with other international experiences.

\section{Conclusions}

This is the first study in the literature in which the ability of NBI cystoscopy to increase the ability to detect suspicious bladder lesions was compared with the use of WL cystoscopy alone in the same patient. The use of NBI cystoscopy, significantly increases our predictive power to identify lesions not visible with WL cystoscopy, especially for unifocal lesions, those $<3 \mathrm{~cm}$ and recurrent lesions. Following WL TURBT, status, dimension and focality are statistically significant determinants in order to detect bladder oncological lesions by NBI cystoscopy rather than WL cystoscopy.

In conclusion, NBI is an effective method for the identification of bladder lesions and can be useful in supporting WL cystoscopy.

\section{REFERENCES}

1. Loidl W, Schmidbauer J, Susani M, et al. Flexible cystoscopy assisted by hexaminolevulinate induced fluorescence: a new approach for bladder cancer detection and surveillance? Eur Urol. 2005; 47:323-6.

2. Grossman HB, Gomella L, Fradet Y, et al. A phase III multicenter comparison of hexaminolevulinate fluorescence cystoscopy and white light cystoscopy for the detection of superficial papillary lesions in patients with bladder cancer. J Urol. 2007; 178:62-7.

3. Fradet Y, Grossman HB, Gomella L, et al. A comparison of hexaminolevulinate fluorescence cystoscopy and white light cystoscopy for the detection of carcinoma in situ in patients with bladder cancer: a phase III, multicenter study. J Urol. 2007; 178:68-73.

4. Denzinger S, Burger M, Walter B, et al. Clinically relevant reduc- tion in risk of recurrence of superficial bladder cancer using 5aminolevulinic acid-induced fluorescence diagnosis: 8-year results of prospective randomized study. Urology. 2007; 69:675-9.

5. Bryan RT, Billingham LJ, Wallace DMA. Narrow-band imaging flexible cystoscopy in the detection of recurrent urothelial cancer of the bladder. BJU Int. 2007; 101:702-6.

6. Kuznetsov K, Lambert R, Rey JF. Narrow-band imaging: potential and limitations. Endoscopy 2006; 38:76-81.

7. Agresti A An introduction to categorical data analysis, $2^{\text {nd }} e d n$, J.Wiley, 2007.

8. Allard P, Bernard P, Fradet Y, et al. The early clinical course of primary $\mathrm{Ta}$ and $\mathrm{T} 1$ bladder cancer: a proposed prognostic index. $\mathrm{Br}$ J Urol. 1998; 81:692-8.

9. Brausi M, Collette L, Kurth K, et al. Variability in the recurrence rate at first follow-up cystoscopy after TUR in stage Ta-T1 transitional cell carcinoma of the bladder: a combined analysis of seven EORTC studies. Eur Urol. 2002; 41:543.

10. Kockelbergh JS. The role of photodynamic diagnosis in the contemporary management of superficial bladder cancer. RC BJU Int. 2005; 96:17-21

11. Witjes JA, Douglass J. The role of hexaminolevulinate fluorescence cystoscopy in bladder cancer. Nat Clin Pract Urol. 2007; $4: 542-549$.

13. Jocham D, Stepp $H$, Waidelich R. Photodynamic diagnosis in urology: State-of-the-art. Eur Urol. 2008; 53:1138-1148.

14. Hamamoto Y, Endo T, Nosho K, et al. Usefulness of narrowband imaging endoscopy for diagnosis of Barrett's esophagus. J Gastroenterol. 2004;39:14-20.

15. Machida H, Sano Y, Hamamoto Y, et al. Narrow-band imaging in the diagnosis of colorectal mucosal lesions: A pilot study. Endoscopy. 2004; 36:1094-1098.

16. Nakayoshi T, Tajiri H, Matsuda K, et al. Magnifying endoscopy combined with narrow band imaging system for early gastric cancer: Correlation of vascular pattern with histopathology (including video). Endoscopy 2004; 36:1080-1084.

17. Shibuya K, Hoshino H, Chiyo M, et al. High magnification bronchovideoscopy combined with narrow band imaging could detect capillary loops of angiogenic squamous dysplasia in heavy smokers at high risk for lung cancer. Thorax. 2003; 58:989-995.

18. Yoshida T, Inoue H, Usui S, et al. Narrow-band imaging system with magnifying endoscopy for superficial esophageal lesions. Gastrointest Endosc. 2004; 59:288-295.

19. Herr HW, Donat SM. A comparison of white-light cystoscopy and narrow-band imaging cystoscopy to detect bladder tumour recurrences; BJU Int. 2008; 102:1111-1114.

\section{Correspondence}

Roberto Giulianelli, MD giulianelli0764@gmail.com

Barbara Cristina Gentile, MD

bcgentile@libero.it

Luca Albanesi, MD

lacalbanesi@hotmail.com

Paola Tariciotti, MD (Corresponding Author)

paola.tariciotti@libero.it

Gabriella Mirabile, MD

gbrmr78@yahoo.com

C.Ur.A., Urology Department, Nuova Villa Claudia Clinic, Rome, Italy 